\title{
SUL PERIODO SISMICO DI PEDARA (ETNA) \\ MANIFESTATOSI NEI PRIMI MESI DEL 1955
}

\author{
Salvature Cucuzza Siltestri
}

Se frequenti e spesso gravi sono i terremoti del basso versante crientale dell'Etna, non meno interessanti sono quelli del versante meridionale, dove, secondo qualche autore (Baratta, De Fiore), si potrebbero addirittura lonalizzare diversi distretti sismici ben distinti.

Con la presente nota viene segnalata una serie di scosse sismiche avvertite, principalmente, durante il mese di Febbraio1955 (ma iniziatasi nei primi giorni del mese di Gennaio, come sarà meglio esposto più avanti) nella zona del comune etneo di Pedara $\left({ }^{*}\right)$.

Secondo alcune informazioni raccolte presso il quartiere alto di Pedara (M. Annunziata). le prime scosse di terremoto sono state avvertite in questa zona il 6 Gennaio a $7^{\mathrm{h}} 30^{\mathrm{m}}$ e a $7^{\mathrm{h}} 45^{\mathrm{m}}$, con intensità fra il II ed il III (**).

Di cuesta orima fase, che potrebbe essere considerata come la fase di apertura del periodo sismico, non si hanno altre notizie.

Presso il Palmento Toscano (2 Kim a N di Pedara sulla strada della Tardaria - quota 780), nello stesso mese di Gennaio, nella notte fra il 18 ed il 19, furono arvertite altre scosse che si ripeterono numerose (circa 9) fra $21^{\mathrm{h}} 3 \mathrm{n}^{\mathrm{m}}$ del 18 e $6^{\mathrm{h}} \mathrm{del} 19$. L'intensità di queste scosse è stata del II-III. Durante la stessa notte a Fleri (fraz. di Zafferana, versante (rientale del vulcano) si verificarono scosse di II-III a $21^{\mathrm{h}} 45^{\mathrm{m}}, 22^{\mathrm{h}}$, $22^{\mathrm{h}} 1 \mathrm{~b}^{\mathrm{m}}, 24^{\mathrm{h}}$ circa e $2^{\mathrm{h}}$ del 19 . Leggermente più forti furono avvertiti a Zafferana diversi tremiti sempre nella stessa notte 18.19. In nessun altro centro della zona si verificarono scosse in tali giorni, nè in quelli immediatamente successivi.

Secondo queste notizie, quindi, all'inizio del periodo sismico di Pedara, si sono avute manifestazioni anche in qualche zona del versante orientale del vulcano, dove, come sarà specificato più avanti, si è solo

(*) Il comune di Pedara è sito a circa $16 \mathrm{~km}$ a S-SE del cratere centrale dell'Etna a quota $610 \mathrm{slm}$.

(**) Per stabilire l'intensità delle scosse si è fatto uso dei gradi della Scala Sismica Mercalli. 


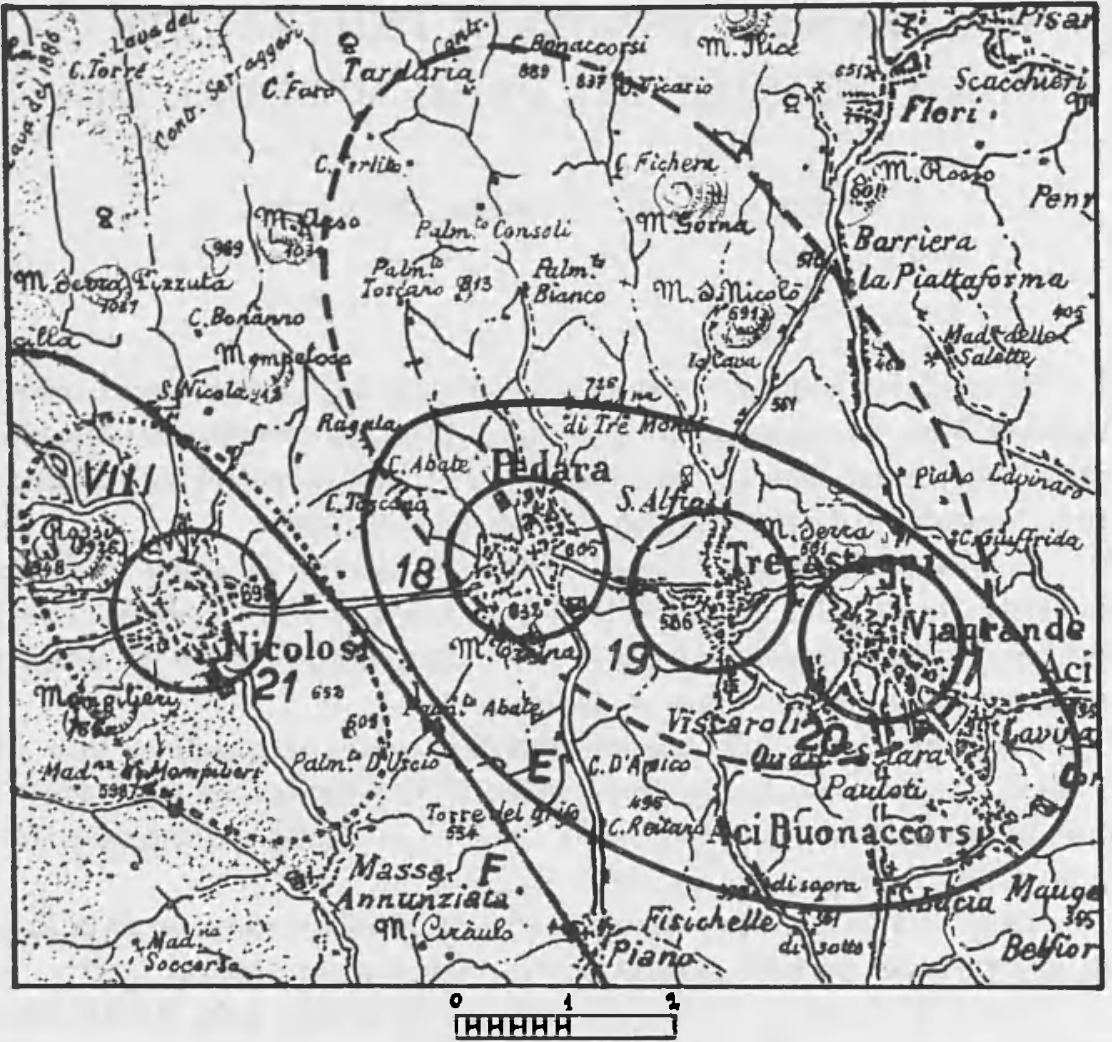

Area mesosismica di VIIl ed isosisma di $V I$ per il terremoto dell'11 Maggio 1901 -secondo IMBO' ( 14 )-

Distretto sismico $E$, parte del distretto $F$ ed aree epic entrali 18, 19, 20 e 21 - secondo De Fiore (5)-

- - Area colpita dal periodo sismico di Pedara del Febbraio 1955 -secondo S. Cucuzza Silvestri - 
notata qualche altra scossa durante il primo gruppo del Febbraio e verso la fine del periodo stesso (*).

Col successivo mese di Febbraio aumentarono il numero e l'intensità delle scosse, che vennero avvertite prinripalmente nel centro di Pedara e nelle campagne circostanti, fino alla Tardaria (frazione sita $4 \mathrm{~km}$ a N del detto centro - a quota 890). Molte di queste scosse vennero notante anche a Trecastagni, poche a Viagrande o pochissime a Fleri.

Il giorno 1 Febbraio a $5^{\mathrm{h}} 25^{\mathrm{m}}$ a Pedare, si avverti una scossa del V, che pare abbia provocato delle piccole lesioni in qualche vecchia casa del centro. Nella serata dello stesso giorno si verificarono numerose scosse leggere, che andarono via via aumentando di numero e di intensità, durante la notte e nelle prime ore del successiro giorno 2, mentre si estese anche l'area interessata. Si fa notare che fra $20^{\mathrm{h}} 45^{\mathrm{m}}$ del giorno 1 e $6^{\text {h }}$ del 2 furono avvertite:

6 scosse nella parte centrale e merid. dell'abitato di Pedara

11 scosse nel quartiere di M. Annunziata

8 scosse nel quartiere di S. Biagio

diverse (non meno di 6) al Palmento Tossano

10 ser:sse alla Tardaria

5 scosse a Trecastagni (Tondo Pedara)

diverse scosse a Trecastagni (centro e quartiere $S$. Alfio)

4-丂̆ scosse a Trecastagni (Gaglianese)

qualche scossa a Viagrande

diverse a Fleri.

Di queste l'intensiti maggiore (V) fu raggiunta a $6^{\text {hi }}$, quando fu investita principalmente la zona Peilara-Tardaria, che può ritenersi l'area epicentrale di questo gruppo di fenomeni sismici. A Trecastagni nessuna scossa superò il IV ed a Viagrande e Fleri il II.

La scossa di $6^{\text {h }}$ provocò un certo panico nella popolazione di Pedara, essendo stata avvertita come forte da molte persone; essa rausó anche dei danni al muro orientale ed al tetto di una casa del paese (via Rimembranze. $81\left({ }^{* *}\right)$.

Date le condizioni della costruzione, piuttosto vecchia e malandata, ritengo che la scossa non abbia raggiunto il VI, ma solo il V.

$\left(^{*}\right)$ Si ba inoltre notizia di due leggere scosse avvertite a $1^{\mathrm{h}} 10^{\mathrm{m}}$ del 7 Maggio a Zafferana.

(**) Questi danni vennero anche segnalati dal Sindaco di Pedara alla Prefettura di Catania. 
Circa la frequenza delle scosse è da tener presente che essa è stata anche notevole durante $\mathrm{i}$ fenomeni sismici che si verificarono successivamente (vedi notte 10-11 Febbraio).

Con la scossa verificatasi a $9^{\mathrm{h}}($ ?) dello stesso giorno 2 Febbraio e con qualche altra leggera notata nella notte anche a Trecastagni, si può considerare finito questo gruppo di scosse.

Ad esso seguirono dei leggeri tremiti isolati o a piccoli gruppi, interessanti la stessa zona, ma su area meno estesa:

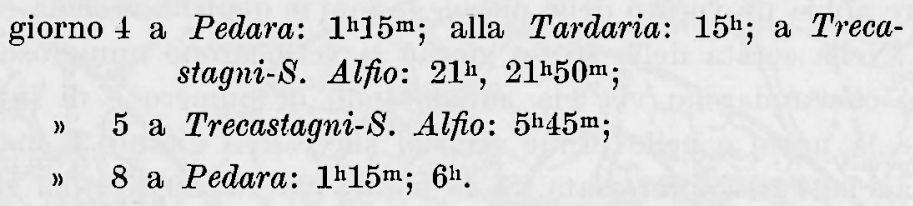

Tutte queste scosse sono state de] III, tranne due o tre del IV (ved. tabella). Esse possono essere considerate come un'appendice del gruppo precedente.

Dopo oltre due giorni ( $5 \tau$ ore) di calma (durante i quali peró sembrerebbe siano state avvertite scosse leggere a Nicolosi) a $15^{\mathrm{h}}$ del 10 Febbraio ricominciarono a verificarsi scosse a Pedara, che si estesero verso Trecastagni a $21^{\mathrm{h}} \mathbf{4}^{\mathrm{m}}$ e, successivamente, durante la notte, con un addensamento tale da determinare addirittura un gruppo dalle caratteristiche analoghe a quelle del gruppo dei giorni 1-2, del quale s'è detto. Infatti il numero delle scosse aumentò piuttosto rapidamente, si allargò verso Est l'area interessata, e il fenomeno, crescendo sempre d'intensità, culminò in commozioni di $\mathrm{V}$, verso le prime ore del giorno $11\left(^{*}\right)$.

Durante la notte $10-11$, vennero avvertite:

$$
\begin{aligned}
& \text { oltre } 5 \text {-scosse a Pedara (centro e periferia) } \\
& \text { oltre } 4 \text { scosse a Trecast.ngni. }
\end{aligned}
$$

Non turono segnalati tremiti nè alla Tardaria (Pellara) nè a $S$. Alfio (Trecastagni), per cui si può ritenere che questo grnppo di scosse sia stato più localizzato, con area epicentrale spostata, rispetto al precedente dei giorni 1-2, verso Sud-Est, essendo stata più colpita la zona Pedara-Tondo di Pedara (Trecastagni).

(*) Della scossa di $V$ delle $5^{\mathrm{h}} 45^{\mathrm{m}}$ ha dato notizia anche il quotidiano di Catania, "La Sicilia " del giorno 12 Febhraio 55, riferendo in generale di altre scosse avvertite, sempre a Pedara, nei giorni precedenti. La scossa non è stata registrata dai sismograf del Collegio "Pennisi " di Acireale (a $\mathrm{km} \mathrm{9,5}$ da Pedara). 
La fase decrescente del gruppo suddetto si protrasse per diversi giorni; essa è rappresentata dalle scosse dello stesso giorno 11 Febbraio a $13^{\mathrm{h}}$, avvertite a Pedara (III-V?) e a Trecastagni (IV); a $15^{\mathrm{h} 30^{\mathrm{m}}}$ ancora a Pedara. Nei successivi giorni 12, 13, 14 si notarono scosse sempre più spostate verso Est (Trecastagni) e verso NE (Fleri): due scosse di II-III fra $15^{\mathrm{h}}$ e $17^{\mathrm{h}}$ del 14 .

Il periodo si chiuse con un gruppo di scosse che si notarono dal 15 al 18 Febbraio, interessanti debolmente Pedara all'inizio (giorno 15 a $5^{\mathrm{h}} 50^{\mathrm{m}}$ : III), piùlintensamente la zona della|Tardaria (notte 16-17: IV-V) ed infine Trecastagni (il 17 a $21^{\mathrm{h}} 16^{\mathrm{m}}$ ed il 18 a $6^{\mathrm{h}} 20^{\mathrm{m}}$, sempre di IV) e Viagrande (notte 17-18: II-III).

Anche in questa fase finale del periodo si osserva lo spostamento dell'area epicentrale sia verso Est e sia verso $\mathrm{N}$, come viene confermato dalle leggere scosse (II-III) notate a Fleri il 14 e nella notte 16-17 contemporaneamente, queste ultime, a quelle più forti (IV-V) della Tardaria.

Raccogliendo tutte le notizie $\left({ }^{*}\right)$ in ordine cronologico, si hanno le tabelle riassuntive riportate nelle pagine che seguono. In esse l'intensità delle scosse è indicata con numeri romani.

Considerando l'andamento dei fenomeni suddetti, si può rilevare quanto segue:

1) Il periodo sismico di Pedara potrebbe dividersi nei seguenti gruppi:

primo gruppo: scosse dei giorni 18 e 19-Gennaio, secondo gruppo: scosse dei giorni 1 e 2-Febbraio, terzo gruppo: scosse dei giorni 10, 11, 12, 13, 14-Febbraio.

A questi gruppi principali, ben distinti nel tempo, sono da collegarsi le scosse delle fasi:

iniziale: del giorno 6 Gennaio;

intermedia fra il sec. ed il terzo gruppo principale: dei giorni 4 , 5, 8 Febbraio;

finale: dei giorni 15, 16, 17 e 18 Febbraio;

(*) Durante i miei numerosi sopraluoghi sono stato coadiuvato dal tecnico dell'Istituto, Sig. Michele Taffara, che ringrazio unitamente a tutti coloro che gentilmente mi hanno fornito notizie e particolari, sia direttamente sia per corrispondenza. 


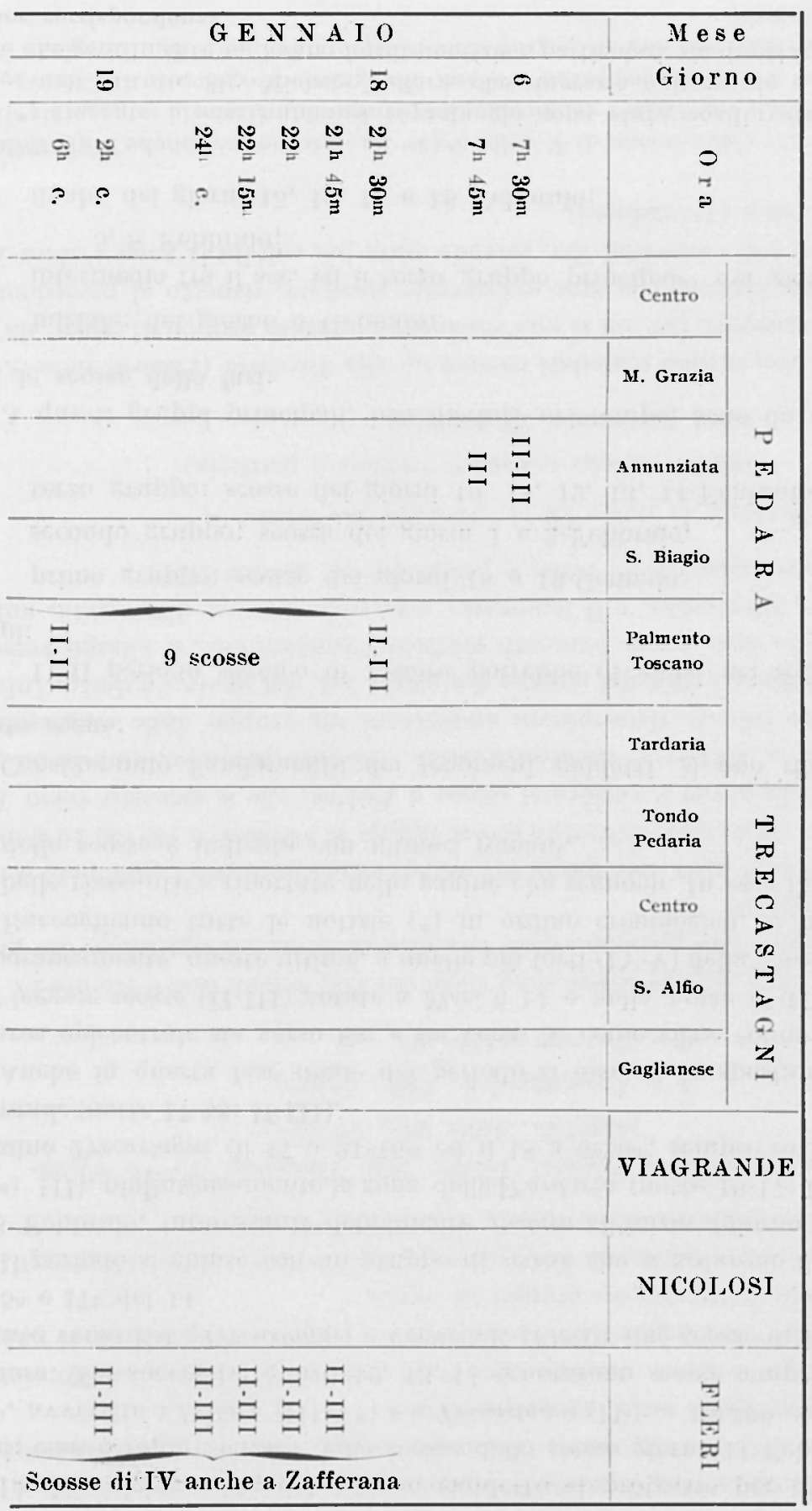




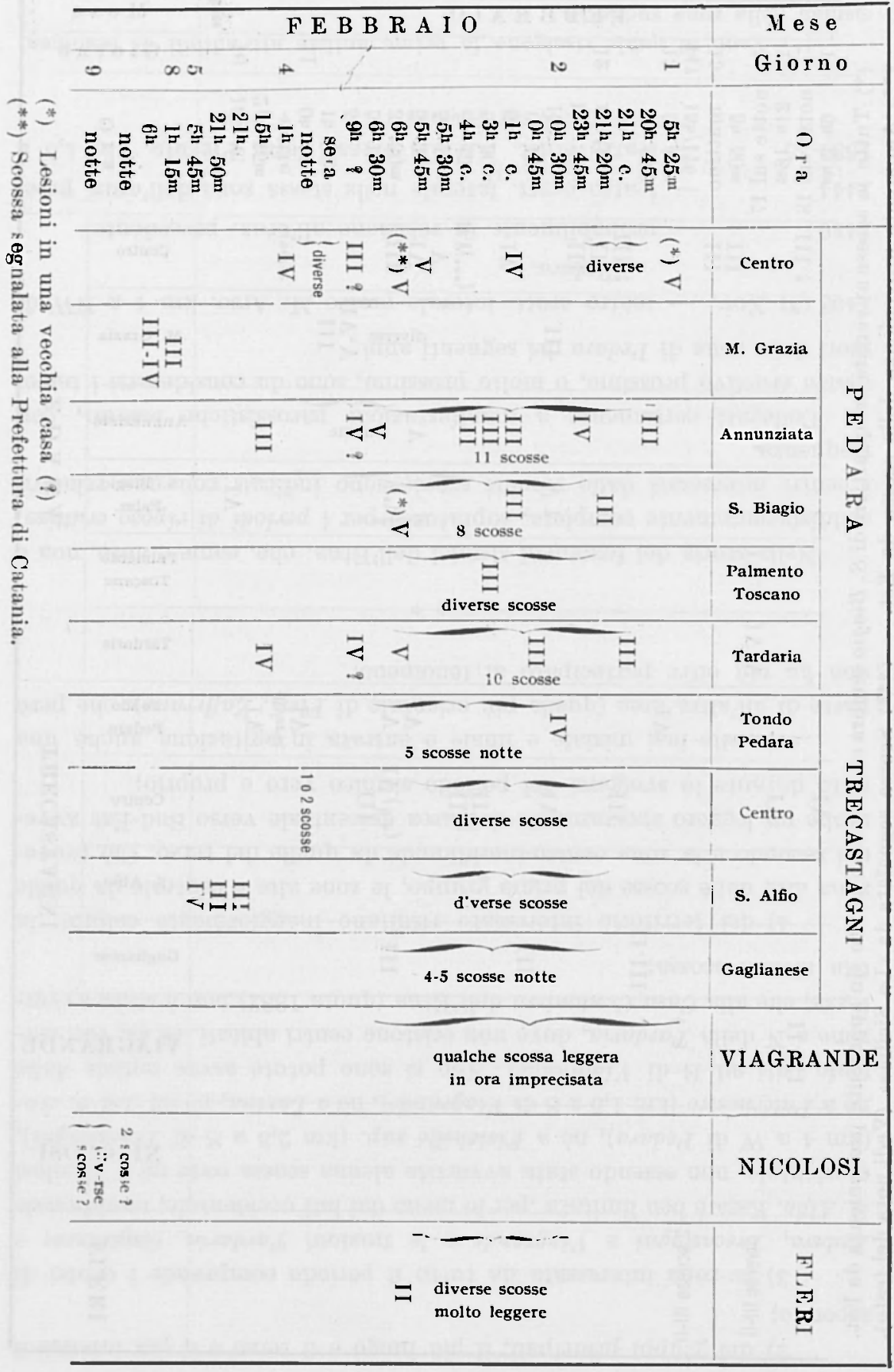




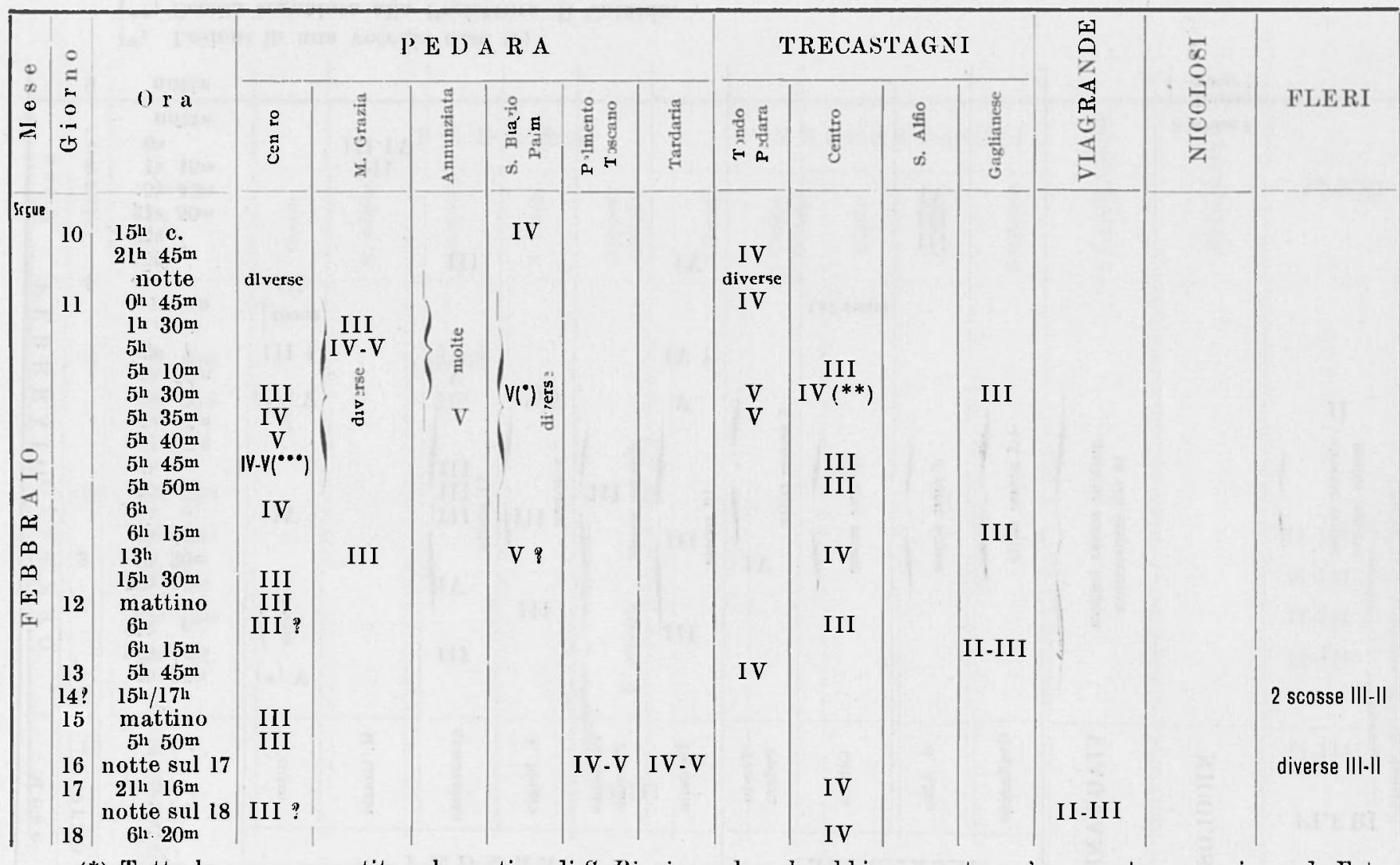

(*) Tutte le scosse avvertite nel quartiere di S. Biagio sembra che abbiano avuto un'apparente provenienza da Est. $(* *)$ Apparente provenienza della scossa da Est. (***) Scossa segnalata da "La Sicilia". (Vedi nota.del testo). 
2) dei gruppi principali, il più lungo è il terzo e il più intenso il secondo;

3) la zona interessata da tutto il periodo comprende i centri di Pedara, Trecastagni e Viagrande e le frazioni Tardaria, Gaglianese e S. Alfio. Essa è ben limitata ,per lo meno dai lati occidentale, meridionale e orientale, non essendo stata avvertita alcuna scossa certa nè a Nicolosi ( $\mathrm{km} 4$ a W di Pedara), nè a Fisichelle sup. (km 2,5 a $\mathrm{S}$ di Trecastagni), nè a Paterncstro ( $\mathrm{km} 1,5 \mathrm{a} \mathrm{S}$ di Viagrande), nè a Lavina, nè ad $A c i-S . A n$ tonio (siti ad E di Viagrande). Non si sono potute avere notizie dalle zone a $\mathrm{N}$ della Tardaria, dove non esistono centri abitati. Si sa, con certezza, che alla Casa Cantoni:rn dell'Etna (quota 1882) non è stata avvertita alcuna scossa;

4) del territorio interessato risultano maggiormente colpite: la zona alta dalle scosse del primo gruppo, le zone alta e centrale da quelle del secondo e la zona centro-meridionale da quelle del terzo. Ciò proverebbe un leggero spostamento dell'area epicentrale verso Sud-Est avvenuto durante lo svolgersi del periodo sismico vero e proprio;

5) nelle fasi iniziale e finale è entrata in agitazione anche una parte di un'altra area (quella più orientale di Fleri, Zafferana), che però non ha più oltre partecipato al fenomeno.

Nella storia dei fenomeni sismici dell'Etna, che, come è noto, non è soddisfacentemente completa, sopratutto per i periodi di riposo eruttivo, i centri interessati dalle recenti scosse sono indicati con una relativa frequenza.

Collegati certamente a manifestazioni parossistiche laterali, con teatro eruttivo prossimo, o molto prossimo, sono da considerarsi i terremoti della zona di Pedara dei seguenti anni:

1408 (*) Nov. - teatro erutt. laterale presso M. Arso. $\mathrm{km} 4$ a NW di Pedara.

1410 - probabilmente in relazione all'eruz. precedente.

1444 - teatro erutt. laterale nella stessa zona dell'eruz. prec.

1534-35 - teatro erutt. laterale presso Serra Pizzuta, km 4,5 a W-NW di Pedrra

(*) Anno al quale risalgono le prime notizie attendibili di fenomeni sismici nella zona suddetta. 

Nicolosi.

1763 Giu. - teatro erutt. laterale della Montagnola, km 12 a N-NW di Pedara.

1883 Mar. - teatro erutt. laterale presso $\mathrm{M}$. Concilio, $\mathrm{km} 7,5$ a NW di Pedura.

Contemporanee a manifestazioni eruttive terminali o subterminali, o, più frequentemente, premonitrici di esse, o, infine, successive ad esse, sono le seguenti scosse che hanno più o meno violentemente colpito la zona di Pedara:

1781 Mag. - che interessarono quasi tutti i centri abitati etnei;

1809 Mar. - che colpirono un'estesa area del vers. $\mathrm{N}$ del vulcano con eruzione laterale del $27-\mathrm{III}$;

1842 Nov. - area colpita molto estesa - versante S:

1906 Giu. - area epicentrale: Massannunziata - attività esplosiva centrale nel Gennaio;

1911 Ott. - area più colpita: Fondo Macchia - eruzione laterale versante $N$ nel Novembre;

1915 Apr. - area colpita: Pedara - contemporanea attività centrale e subterminale;

1916 Dic. - idem;

1921 Gen. - area più colpita Zafferana - successiva attività subterm. nel Marzo;

1923 Nov. - area colpita estesa verso Sud Est - eruzione laterale nel Giugno-Luglio versante $\mathbf{N}$;

1931 Ago. - area più colpita: versante $\mathrm{E}$ - ripresa attività centrale in Luglio.

Da questi due elenchi si rileva che in cinque secoli e mezzo la zona di Pedara è stata colpita da 17 terremoti - o periodi sismici - certamente riferibili ad eruzioni laterali (7) o ad attività eruttiva centrale e subterminale (10). Vedi, in fine, le conclusioni riassuntive al n. 6.

Questa constatazione potrebbe essere considerata di notevole interesse, giacchè, come è dimostrato più avanti — sempre attraverso notizie storiche attendibili di macrosismi - quella di Pedara rappresenta 
una zona di relativa stabilità, le cui rare ed eccezionali vibrazioni svelerebbero - con buona probabilità - un risveglio del dinamismo eruttivo etneo.

Nel considerare la distribuzione dei terremoti del basso versante meridionale dell'Etna e tenendo presente le caratteristiche di quelli che hanno colpito prevalentemente la zona di Pedara, si nota che detta zona generalmente non partecipa alle vibrazioni, anche violentissime, del vicino centro di Nicolosi (ad appena $4 \mathrm{~km}$ di distanza). Ricordo infatti i terremoti avvertiti appunto a Nicolosi, e non a Pedara, nei seguenti anni:

1663 - i cui effetti furono disastrosi nella contrada Piano del citato paese, con gravi danni e 17 morti;

1766 - periodo simico collegato allo sgorgo lavico verificatosi a $\mathrm{S}$ della Montagnola;

1805 (3 Lug.) - in relazione ad attività centrale del vulcano;

1818 (20 Feb.) - violentissimo sisma dalla vasta ed irregolare area epicentrale non comprendente nè Pedara, nè Trecastagni, nè Viagrande;

1832 (24-26 Nov.) - in relazione all'eruz. lat. di Bronte:

1852 - contemporanei all'eruz. lat. in Falle d. Bove (20-21-VIII 1852 $27-\mathrm{V}-1853)$;

1885 - periodo sismico del Set.-Ott. con scosse anche di VIII. in area molto circoscritta;

1892 (Lug.) - in relazione all'eruz. lat. dei M. Silvestri, con scuotimenti di quasi tutto l'edificio etneo;

1893 (1 Apr.) - avvertito del VI a Zafferana...:

1894 (7 Ago.) - avvertito del VIII a Zerbate...;

1894 (8 Ago.) - avvertito del IX a Mazzasette.F'leri ...;

1897 (Feb.) - avvertito del V a Riposto, Mascalucia ...:

1898 (Mag.) - avvertito dell'VIII a Ragalna ...;

1900 (Ott.) - locale: Nicolosi;

1902 (Feb.) - avvertito del V a Zafferana ...;

1903 (Gen.) - avvertito del V a Zafferana, Milo ...;

1903 (Mar.) - avvertito del Y a Massannunziata ...; 
1903 (Nov.) - avvertito del VI a Viagrande, Zafferana, Milo ...;

1905 (Mag. \& Set.) - avvertito de] V a Milo, Zafferana, Randazzo...; 1907 (Lug. e Dic.) - avvertito dell'VIII a Zerbate e del VI a Zaffe-

$$
\text { rana...; }
$$

1908 (Mag. e Dic.) - avvertito del VI a Milo ...;

1914 (Apr. e Mag.) — periodo sismico interessante prevalentemente i versanti $\mathrm{N}$ ed $\mathrm{E}$ :

1917 (Giu.) - avvertito del VI a Ragalna ...;

1918 (Giu.) - avvertito del VI a Biancavillu ...;

1919 (Mag.) - locale Nicolosi;

1928 (Mar.) - (ricordo l'eruz. laterale del Novembre).

La zona di Pedara presenta inoltre una certa indipendenza dai fenomeni sismici verificatesi anche in altre zone dello stesso versante meridionale del vulcano e di quello orientale. Già ho segnalato fra i terremoti che hanno colpito Nicolosi e risparmiato Pedara, quelli che non hanno avuto Nicolosi stesso come area epicentrale (*); a questi aggiungo le seguenti scosse manifestatesi in altre zone anche vicine o vicinissime senza colpire Pedara:

2 Ott. 1909 (III a Viagrande. VII a Malati);

15 Mag. 1910 (III a Viagrande);

20 Gen. 1911 (III a Viagrande);

4 Ott. 1912 (III a Trecastagni, VI a Milo);

25 Giu. 1913 (III a Viagrande, V a .Milo);

19 Ago. 1913 (II a Trecastagni, V a Biancavilla);

Mag. 1914 (periodo sismico interessante il versante orientale, con scosse di IV-V-VII a Viagrande);

Giu. e Nov. 1920 (II a Viagrande, isolate, locali);

1 Feb. 1932 (deboli a Trecastagni, isolate);

$\left(^{*}\right)$ Cioè quelli degli anni 1766, 1818, 1832, 1892, 1893, 1894, 1897, $1898,1902,1903,1905,1907,1908,1914,1917,1918$. 
13 Apr. 1938 (VI a Viagrande);

1 Mar. 1952 (III a Viagrande - collegate al violento periodo sismico di Princessa Zafferana).

Indipendenti da attività eruttiva sono da considerarsi soltanto $i$ seguenti terremoti della zona di Pedara:

- quello. locale, proprio di Pedara (IV), del Nov. 1898 (arvertito di II soltanto a Biancavilla);

- il periodo sismico di Nicolosi (scosse fino all' VIII) del Mag. 1901, che colpì anche Pedara con scosse del V, Trecastagni (III) e Viagrande $(\mathrm{II})$.

Attraverso i dati che ho esposto si possono trarre le seguenti conclusioni:

1) il periodo sismico oggetto della presente nota. ha interessato una zona abbastanza circoscritta, attorno al centro di Pedara, con le caratteristiche, già riassunte, le quali ricordano i terremoti degli anni 1634, 1915, e 1916:

2) i terremoti della zona di Pedara generalmente investono un'area abbastanza circoscritta;

3) le scosse non si propagano che molto raramente in zone vicine; se ciò si verifica, l'eccitamento si ha principalmente verso Est e verso Nord Est;

4) le scosse non sono mai molto forti (con massimi d'intensità intorno al V-VI);

5) le scosse di altre zone, anche vicine, difficilmente provocano la vibrazione della regione di Pedara (vedi indipendenza già dimostrata dai sismi di Nicolosi e da quelli del versante orientale del vulcano);

6) generalmente l'attività sismica della regione oggetto di questa nota è da collegarsi ad una ripresa eruttiva del vulcano già manifestatasi immediatamente prossima nel tempo e nello spazio (specie se eruzioni laterali), ovvero con fenomeni prarossistici terminali e subterminali (anche a distanza di qualche mese); 
7) il numero e la frequenza dei terremoti registrati nella zona di Pedara non sono tali da far considerare detta zona come un'area sismica vera e propria, contrariamente a quanto ha asserito qualche autore (*).

8) le scosse dei centri vicini colpiscono la zona di Pedara soltanto se violente e frequenti, ciò che dimostra una certa stabilità della zona stessa;

9) questa stabilità viene turbata isolatamente soltanto quando abbiano a verificarsi "rari " assestamenti superficiali (postumi d'eruzione, etc.) o movimenti magmatici non molto profondi;

10) la recente attività sismica può essere infatti riferita a fenomeni del suddetto ultimo tipo, come dimostra la seguente tabella riassuntiva dell'attività centrale dell'Etna nell'anno $1955\left({ }^{* *}\right)$, dalla quale si ricava che a circa due mesi dal periodo sismico di Pedara è cominciato il risveglio subterminale del vulcano.

(*) Il Baratta (') comprende Pedara e Trecastagni nella regione sismica di Nicolosi, cioè in quella zona interessata da un certo numero di scosse che avrebbero avuto "il focolare situato nei pressi di Nicolosi ". Egli, però, nel descrivere i fenomeni sismici della regione, accenna tanto a scosse localissime, avvertite, spesso con notevoli danni, nel solo centro di Nicolosi, quanto a scosse più estese, segnalate nei centri vicini, per cui non appare ben caratterizzata e distinta questa presunta regione sismica.

Nelle relazioni isolate dei terremoti etnei prima e dopo della citata pubblicazione del Baratta (Silvestri, Arcidiacono, Ricco, Platania, etc.) non si riscontrano distinzioni di zone sismiche, distretti, aree che possono interessare la regione di Pedara.

Bisogna arrivare ai lavori conclusivi del De Fiore, prima, e dell'Imbò, dopo, per avere sistematiche e particolareggiate ricerche sulla sismicità etnea e sulla distribuzione delle aree epicentrali.

Il primo A. infatti ammette l'esistenza di aree sismiche ben definite $e$ sicure (alle quali corrisponde almeno un ipocentro ben distinto). Fra queste aree egli inserisce Trecastagni e Tiagrande $\left(^{(4)}\right.$ e successivamente Pedara $\left({ }^{5}\right)$. Lo stesso A. inoltre comprende questi tre centri in un distretto sismico indipendente da quello più occidentale di Nicolosi.

L'Imbò (14) distingue in tutta l'Etna tre zone sismiche "entro le quali sarebbero situati gli ipocentri ".

In tal modo la regione compresa tra Nicolosi e Trescatagni apparterebbe alla area meridionale e Viagrande invece rimarrebbe fra questa e quella orientale, senza essere considerata un vero e proprio epicentro.

(**) Più ampi particolari si trovano nella relazione da me fatta in altra nota sui più recenti fenomeni eruttivi dell'Etna. 
Attività Centrale E subterminale Dell'Etxa xell'año 1955

\begin{tabular}{|c|c|c|}
\hline Gennaio & & $\begin{array}{l}\text { esalazione lenta di vapori bianchi dalla sola voragine } \\
\text { del cratere subterminale di Nord Est; continuano } \\
\text { a verificarsi esplosioni "profonde ", senza proie- } \\
\text { zione di materiale solido fuori della voragine; non } \\
\text { si notano bagliori; }\end{array}$ \\
\hline $\begin{array}{l}\text { Febbraio- } \\
\text { Marzo }\end{array}$ & & $\begin{array}{l}\text { esalazione lenta di vapori bianchi (qualche giorno con } \\
\text { violenti "sbuff "); }\end{array}$ \\
\hline \multirow[t]{4}{*}{ Aprile } & 5 & $\begin{array}{l}\text { bagliori notturni, segnalati da Taormina (da } 20^{\mathrm{h}} \text { a } \\
20^{\mathrm{h}} 30^{\mathrm{m}} \text { ) segno di affioramento di magma nel fondo } \\
\text { del cratere subterminale di Nord Est; }\end{array}$ \\
\hline & $26-27$ & $\begin{array}{l}\text { sabbia grigia non magmatica, caduta a Riposto nella } \\
\text { notte, proveniente probabilmente dal cratere } \\
\text { subterminale; }\end{array}$ \\
\hline & 27 & $\begin{array}{l}\text { boati segnalati da Zafferana (da } 13^{\text {h }} \text { a } 13^{\mathrm{h}} 30^{\mathrm{m}} \text { ) - segno } \\
\text { probabile di una breve attività esplosiva del era- } \\
\text { tere subterminale di } \mathrm{NE} \text {; }\end{array}$ \\
\hline & 28 & $\begin{array}{l}\text { si nota cenere scura caduta attorno all'orlo del cratere } \\
\text { subterminale di } \mathrm{NE} \text { (?); }\end{array}$ \\
\hline \multirow[t]{2}{*}{ Maggio } & 4 & $\begin{array}{l}\text { bagliori con lancio di scorie incandescenti fuori dell'orlo } \\
\text { della voragine del cratere subterminale di NE (in- } \\
\text { torno a } 20^{\mathrm{h}} \text {; }\end{array}$ \\
\hline & 5 & $\begin{array}{l}\text { emissione di vapori grigi contenenti sabbia magmatica } \\
\text { dal cratere subterminale di NE; lunghi intervalli } \\
\text { di esalazione di soli vapori bianchi; }\end{array}$ \\
\hline \multirow[t]{2}{*}{ Giugno } & & $\begin{array}{l}\text { questa attività dura per quasi tutto il mese; } \\
\text { attività subterminale apparentemente ridotta, con } \\
\text { esplosioni "profonde " e con esalazione di no- } \\
\text { tevoli masse di vapori; }\end{array}$ \\
\hline & 29 & $\begin{array}{l}\text { inizia l'attività parossistica subterminale che culmina } \\
\text { in quella centrale del settembre; }\end{array}$ \\
\hline $\begin{array}{l}\text { Ottobre- } \\
\text { Dicembre }\end{array}$ & & $\begin{array}{l}\text { continua e si esaurisce l'attività parossistica subter- } \\
\text { minale, mentre il cratere centrale entra in fase di } \\
\text { lenta esalazione fumarolica. }\end{array}$ \\
\hline
\end{tabular}

Istituto di Vulcanologia dell'Università di Catania, 19.5.5.

\section{RIASSUNTU}

L'autore si sccupa dello studio di alcuni terremoti verificatisi nei mesi di Gennaio e Febbraio 19.5.j nella zona di Pedara, versante meridionale dell'Etna. Le caratteristiche di questi fenomeni sismici rengono confrontate 
con quelle di fenomeni analoghi verificatisi nel passato. Se ne ricava che $i$ terremoti di Pedara:

- sono estremamente localizzati in 'area 'abbastanza circoscritta al centro abitato:

- sono poco violenti ( $V-I V)$;

- non provengono generalmente da agitazioni sismiche di zone vicine o vicinissime (es. Nicolosi);

- non sono frequenti (tanto che la zona non ha specifici caratteri di "Marea sismica").

Sui dati macrosismici passati e considerando l'andamento dei fenomeni eruttivi etnei - anche attuali — si avanza l'idea che la sismicità della zona sia collegata principalmente all'attività eruttiva del vulcano, forse più di quanto non sia constatato per altre zone dell'Etna.

\section{B I B I I O G R A F I A}

(1) Baratta, M., I terremoti d'Italia. Torino, 1901.

$\left({ }^{2}\right)$ - I terremoti in Italia. Pubbl. Commissione It. studio grandi calamità, Vol. VI, Firenze, 1936.

( ${ }^{3}$ Comin, G., Il terremoto di Codavolpe (vers. orientale Etna) dell'8-IV-1950. Boll. Acc. Gioenia, Ser. IV, Vol. II. fasc. 8, Catania, 1954.

(') - Su l'attività eruttiva terminale dell'Etna iniziatasi il 2.5 giugno 1955. "Stromboli ", N. 4, 1955.

$\left(^{5}\right)$ De Frore, O., Il periodo di riposo dell'Etna 1893-1907. Mem. Acc. Zelanti Ser. III, Vol. VI. Acireale, 1908-09.

${ }^{(8)}$ - L'Etna. Il "periodo di riposo" 1911-18. Catania, 1919.

(7) - Come è stato e come dovrebbe essere studiato l'Etna. Catania 1919.

${ }^{(8)}-I$ fenomeni eruttivi, sismici e pseudovulcanici avvenuti all'Etna nel 1916. Boll. Soc. Sismol. It. Vol. XXI-XXIV. Anni 1917-18, 1922-23. Modena, 1924.

${ }^{\left({ }^{8}\right)}$ - I fenomeni eruttivi e sismici avvenuti all'Etna dal 1919 al 1923. Ann. Osservatorio Vesuviano, Ser. IlI, vol. II, Napoli, 1925.

${ }^{\left({ }^{10}\right)}-I$ movimenti tellurici (del $1 I I-1952$ ). Intervista col Direttore dell'Ist. Vulcanologico Etneo. "Il Corriere" di Catania del 4-III-1952.

(11) Gemeltaro, C., La Vulcanologia dell'Etna. Atti Acc. Gioenia, Ser. II, Vol. XIV-XV, Catania, 1858-59.

$\left.{ }^{12}\right)$ Iмвó, G., Terremoti della regione orientale etnea. Bull. Volcanologique, N.ri 11-18, Napoli, 1928.

${ }^{\left({ }^{13}\right)}$ - L'attività eruttiva e sismica dell'Etna dal Nov. 1928 a tutto il 1923. Annali Osserv. Vesuviano, Ser. IV, Vol. II, Napoli, 1929-30. 
(14) Iмвò, (i., Linee isosismiche in zone vulcaniche con particolare riguardo all Ema. Mem. Ace. Zelanti, Ser. IV, Vol. III, Acireale.

${ }^{(15)}$ - I terremoti etnei. Pubbl. Commissione It. studio grandi calamitì. Vol. V, parte 1, Firenze, 1935.

(16) Platania, G., Sul periodo sismico del maggio 1914 nella regione orientale dell'Etna. Mem. Acc. Zelanti, Ser. III, Vol. VII, Acireale 1915.

(17) - Sul periodo sismico del Nov. 1919 presso Acireale. Boll. Osserv. Geodinamico di Catania, Nuova Ser., n. 3-4, 1920.

(18) Sartorius von Waltershausex, W., Der Aetha. Leipzig, 1880. 